\title{
Effects of Spatial Curvature on the $f(R)$ Gravity Phase Space: no Inflationary Attractor?
}

\author{
S. D. Odintsov, ${ }^{1,2}$ V.K. Oikonomou, ${ }^{3,4,5}$ \\ 1) ICREA, Passeig Luis Companys, \\ 23, 08010 Barcelona, Spain \\ 2) Institute of Space Sciences (IEEC-CSIC) C. Can Magrans $s / n$, \\ 08193 Barcelona, Spain \\ 3) Department of Physics, \\ Aristotle University of Thessaloniki, \\ Thessaloniki 54124, Greece \\ 4) Laboratory for Theoretical Cosmology, \\ Tomsk State University of Control Systems and Radioelectronics, \\ 634050 Tomsk, Russia (TUSUR) \\ 5) Tomsk State Pedagogical University, \\ 634061 Tomsk, Russia
}

\begin{abstract}
In this paper we study the effects of spatial curvature of the metric on the phase space of vacuum $f(R)$ gravity. Particularly, we appropriately choose the variables of the dynamical system, in order for this to be autonomous, and we study the phase space of the resulting theory, focusing on de Sitter, matter and radiation domination fixed points. Our analysis indicates that the effect of spatial curvature on the phase space is radical, since it destabilizes all the stable de Sitter vacua of the flat spacetime vacuum $f(R)$ gravity phase space, making the phase space having non-trivial unstable submanifolds. This instability occurs regardless if the spacetime has elliptic or hyperbolic spatial sections, and it is also robust towards the choice of initial conditions. We investigate the source of the instability in the system, and also we discuss the stability of the matter and radiation domination vacua, which, as we demonstrate, are also highly unstable. Our results for de Sitter attractors indicate that the stable de Sitter attractors of the vacuum $f(R)$ gravity theory for a flat Universe, are destabilized by the presence of curvature, and this shows that inflation for vacuum $f(R)$ gravity in non-flat spacetime is problematic, at least at the phase space level. This result holds true for both elliptic and hyperbolic spacetimes.
\end{abstract}

PACS numbers: 95.35.+d, 98.80.-k, 98.80.Cq, 95.36.+x

\section{INTRODUCTION}

Among the various forms of modified gravity, $f(R)$ gravity provides the simplest and most elegant to our opinion, description of several evolutionary aspects of our Universe [1 6]. Indeed, it is possible to describe two of the most mysterious acceleration eras of our Universe, namely the inflationary and the late-time acceleration era, in a unified framework [7, 8], and several works in the literature investigate the astrophysical and cosmological implications of $f(R)$ gravity, for an important stream of viable $f(R)$ gravity models, see for example [7, 9 24]. In view of the importance of $f(R)$ gravity in modern cosmology applications, it is vital to know the general behavior of $f(R)$ gravity in a model independent way, and the only formal and mathematically rigid way to achieve this is to study the phase space of $f(R)$ gravity. The correct approach for doing so, is to construct an autonomous dynamical system for the simplest $f(R)$ gravity action, namely that of vacuum $f(R)$ gravity. Actually the dynamical system approach is frequently adopted in the literature for studying cosmological systems with cosmological applications in the study of inflation and dark energy for modified gravity and scalar-tensor theories and cosmological fluids, see for example [25 66]. The autonomous dynamical system approach was adopted in several previous works on $f(R)$ gravity [58] and other modified gravities [57, [59], for the study of de Sitter eras and also for matter domination eras. To our opinion, the autonomous dynamical system approach is the most correct approach in studying dynamical systems, since if a dynamical system is non-autonomous, then the linearization techniques, which hold true for autonomous dynamical systems, like the Hartman-Grobman theorem, may lead to mathematical inconsistencies and wrong results. For example [67], the dynamical system $\dot{x}=-x+t$ has an explicit solution $x(t)=t-1+e^{-t}\left(x_{0}+1\right)$, hence all the solutions corresponding to various initial conditions, asymptotically approach $t-1$. However, if one applies the linearization theorems, one finds that the fixed point is time dependent and it is $x=t$, which is not even a solution to the initial dynamical system. In our previous work on vacuum $f(R)$ gravity autonomous dynamical system [58], the whole study was performed by assuming a spatially flat Friedmann-Robertson-Walker (FRW) geometric background. However due to the fact that there exist several studies which indicate that a spatially curved and actually closed 
$\Lambda$ CDM model, provide better fit to the low multipole CMB data $[68,69]^{1}$, in this work, we will concretely examine the effects of spatial curvature on the phase space corresponding to the autonomous dynamical system of vacuum $f(R)$ gravity. In the literature there exist many works focusing on the effects of spatial curvature on the phase space, see for example [25, 63 66]. The focus will be on the existence and stability of de Sitter vacua and also for other possible attractor solutions, like matter and radiation domination attractors. Our study will be focused on the first $60-70$ $e$-foldings, which is a characteristic time interval for inflationary attractors. In most of the cases, the presence of the spatial curvature will make the phase space quite more complicated, so we shall rely to numerical analysis in order to examine the behavior of the trajectories in the phase space. As in the vacuum $f(R)$ gravity in flat FRW spacetime, the time-dependence in the dynamical system is contained in the parameter $m=-\frac{\ddot{H}}{H^{3}}$, so by assuming that this parameter is constant, we examine a subclass of the phase space which can actually yield such a value for $m$, and we seek for stable regions or fixed points for this subclass of $f(R)$ gravity cosmologies. In contrast to the flat vacuum $f(R)$ gravity autonomous dynamical system, the spatially curved autonomous $f(R)$ gravity dynamical system has no stable de Sitter attractors, nor has stable attractors of matter and radiation domination type. As we will demonstrate in our detailed analysis, spatial curvature induces strong instabilities in the phase space. This result clearly indicates that the stable inflationary attractors of the flat vacuum $f(R)$ gravity cease to be stable in the non-flat vacuum $f(R)$ gravity, regardless if the curvature is positive (elliptic spatial sections) or negative (hyperbolic spatial curvatures). This result can be interpreted that inflation for $f(R)$ gravity in non-flat spacetimes seems to be problematic.

This paper is organized as follows: In section I we review the properties of the flat space autonomous dynamical system of vacuum $f(R)$ gravity, emphasizing on the de Sitter fixed points and their stability. In section II, we construct the non-spatially flat autonomous dynamical system of vacuum $f(R)$ gravity, and we investigate the existence of de Sitter, matter domination and radiation domination fixed points. We examine the stability of the fixed points and we compare the non-flat dynamical system with the flat dynamical system. Also we solve numerically the dynamical system and investigate the behavior of the trajectories in the phase space as a function of the $e$-foldings number $N$, emphasizing in values in the range $N=[0,60]$. Finally the conclusions follow in the end of the paper.

\section{AUTONOMOUS DYNAMICAL SYSTEM OF VACUUM $f(R)$ GRAVITY IN FLAT SPACETIME: AN OVERVIEW}

Before we get to the core of this paper, it is worth highlighting the most important outcomes of the flat autonomous $f(R)$ gravity dynamical system study, and for details we refer the reader to Ref. [58]. The general vacuum $f(R)$ gravity action is,

$$
\mathcal{S}=\frac{1}{2 \kappa^{2}} \int \mathrm{d}^{4} x \sqrt{-g} f(R)
$$

with $\kappa^{2}=8 \pi G=\frac{1}{M_{p}^{2}}$ and in addition $M_{p}$ stands for the Planck mass scale. In this work we shall adopt the metric formalism, so by varying the action (1) with respect to the metric $g_{\mu \nu}$, we get the equations for motion for vacuum $f(R)$ gravity,

$$
F(R) R_{\mu \nu}(g)-\frac{1}{2} f(R) g_{\mu \nu}-\nabla_{\mu} \nabla_{\nu} F(R)+g_{\mu \nu} \square F(R)=0
$$

which can be rewritten,

$$
R_{\mu \nu}-\frac{1}{2} R g_{\mu \nu}=\left(\frac{1}{F(R)}\left(\frac{f(R)-R F(R)}{2} g_{\mu \nu}+\nabla_{\mu} \nabla_{\nu} F(R)-g_{\mu \nu} \square F(R)\right)\right),
$$

where the prime indicates differentiation with respect to $R$, the Ricci scalar. The flat FRW metric has the following line element,

$$
d s^{2}=-d t^{2}+a(t)^{2} \sum_{i=1,2,3}\left(d x^{i}\right)^{2}
$$

where $a(t)$ is the scale factor, and the corresponding Ricci scalar is,

$$
R=6\left(\dot{H}+2 H^{2}\right)
$$

${ }^{1}$ However high multipole data support the flat $\Lambda$ CDM. 
where $H=\frac{\dot{a}}{a}$ is the Hubble rate. By applying the FRW metric in (3), the equations of motion take the following form,

$$
\begin{aligned}
& 0=-\frac{f(R)}{2}+3\left(H^{2}+\dot{H}\right) F(R)-18\left(4 H^{2} \dot{H}+H \ddot{H}\right) F^{\prime}(R), \\
& 0=\frac{f(R)}{2}-\left(\dot{H}+3 H^{2}\right) F(R)+6\left(8 H^{2} \dot{H}+4 \dot{H}^{2}+6 H \ddot{H}+\dddot{H}\right) F^{\prime}(R)+36(4 H \dot{H}+\ddot{H})^{2} F^{\prime}(R),
\end{aligned}
$$

with $F(R)=\frac{\partial f}{\partial R}, F^{\prime}(R)=\frac{\partial F}{\partial R}$, and $F^{\prime \prime}(R)=\frac{\partial^{2} F}{\partial R^{2}}$. We can form an autonomous dynamical system for the vacuum $f(R)$ gravity, by using the following dimensionless variables,

$$
x_{1}=-\frac{\dot{F}(R)}{F(R) H}, \quad x_{2}=-\frac{f(R)}{6 F(R) H^{2}}, \quad x_{3}=\frac{R}{6 H^{2}},
$$

so by using the $e$-foldings number instead of the cosmic time, as a dynamical variable that quantifies the evolution, after some algebra we obtain the following dynamical system for the flat vacuum $f(R)$ gravity,

$$
\begin{aligned}
& \frac{\mathrm{d} x_{1}}{\mathrm{~d} N}=-4+3 x_{1}+2 x_{3}-x_{1} x_{3}+x_{1}^{2}, \\
& \frac{\mathrm{d} x_{2}}{\mathrm{~d} N}=8+m-4 x_{3}+x_{2} x_{1}-2 x_{2} x_{3}+4 x_{2}, \\
& \frac{\mathrm{d} x_{3}}{\mathrm{~d} N}=-8-m+8 x_{3}-2 x_{3}^{2},
\end{aligned}
$$

where we used Eqs. (8) and (6), and in addition, the following differentiation rule,

$$
\frac{\mathrm{d}}{\mathrm{d} N}=\frac{1}{H} \frac{\mathrm{d}}{\mathrm{d} t}
$$

Moreover, the parameter $m$ appearing in Eq. (9) is equal to,

$$
m=-\frac{\ddot{H}}{H^{3}},
$$

so the only time dependence (or equivalently $N$-dependence) is contained in the parameter $m$. Therefore, in the cases that $m=$ constant, the phase space solutions are narrowed down and the dynamical system (9) becomes autonomous. It is conceivable that when we specify the parameter $m$ to be constant, in some sense we seek for a class of cosmological solutions of a specific form, hence by studying the dynamical system (9) for the given value of $m$, clearly reveals whether the vacuum $f(R)$ gravity theory can realize such a class of solutions, and also indicates the behavior of the trajectories in the phase space, or equivalently, such a study reveals the existence and stability of cosmological fixed points in the vacuum $f(R)$ gravity phase space. In the flat vacuum $f(R)$ gravity case, the equation of state (EoS) parameter $w_{e f f}$, which is in general defined to be,

$$
w_{e f f}=-1-\frac{2 \dot{H}}{3 H^{2}},
$$

when expressed in terms of the variables (8), can be written as follows,

$$
w_{e f f}=-\frac{1}{3}\left(2 x_{3}-1\right) \text {. }
$$

In Ref. [58] we focused our analysis in the cases $m=0$ and $m=-\frac{9}{2}$, which correspond in the de Sitter and matter domination cases. Note that the quasi-de Sitter evolution $a(t)=e^{H_{0} t-H_{i} t^{2}}$, along with the exact de Sitter evolution $a(t)=e^{\Lambda t}$, can yield $m=0$, but these are not the only cosmologies that may yield $m=0$, for example the symmetric bounce may also yield $m=0$. The nature of the attractors of the phase space for $m=0$ will be determined solely by the value of the effective EoS parameter $w_{\text {eff }}$ at the resulting fixed point. At this point we need to further clarify the case $m=$ const, focusing for example on the case $m=0$. By solving the differential equation (11) for $m=0$ one obtains the quasi-de Sitter solution $H(t)=H_{0}-H_{i} t$, which is indeed an inflationary solution known to provide viable cosmological solutions in $f(R)$ gravity. However, our strategy is to assume that $m=0$ without solving explicitly the differential equation (11). As we already mentioned, there are many cosmologies that may yield $m=0$ apart from 
the quasi-de Sitter one, for example the symmetric bounce $a(t)=e^{-\Lambda t}$, in which case $H(t)=-2 \Lambda t$, and the bouncing point might be the time instance $t=0$. In that case, the solution for the Hubble rate is of the form $H(t)=H_{0}-H_{i} t$, but with $H_{0}$ being set equal to zero. So the case $m=0$ describes a class of cosmological models, not a specific one. Now by setting $m=0$, and by studying the behavior of the dynamical system, one may reveal what is the nature of the cosmological solution, by finding the fixed points and evaluating for these the EoS parameter $w_{e f f}$ given in Eq. (13). This is exactly what we did in Ref. [58], where we demonstrated that the case $m=0$ in the flat space $f(R)$ gravity dynamical system, leads to fixed points which have $w_{e f f}=-1$. Thus the dynamical system indicated directly what is the nature of the subclass of cosmological solutions corresponding to $m=0$. The method is powerful and formal since we do not fix the form of the Hubble rate, by specifying the initial conditions for example (that is we do not fix $H_{0}$ and $H_{i}$ in the solution $\left.H(t)=H_{0}-H_{i} t\right)$, and the dynamical system analysis reveals the nature of the resulting attractor solution, by yielding $w_{\text {eff }}=-1$, which is an exact de Sitter final attractor. Also it is important to note that the system may start from a quasi-de Sitter solution, and it results to an exact de Sitter solution, as was demonstrated in Ref. [58]. Thus the method of dynamical systems reveals the actual evolution of the cosmological solution without making any specific assumptions on the initial conditions chosen for the Hubble rate, apart from the obvious choice of the subclass of solutions corresponding to $m=0$. In conclusion, one does not specify the exact value of the Hubble rate by setting $m=0$, but a subclass of cosmological solutions is studied. The dynamical system analysis reveals the exact evolution in terms of the fixed points and the corresponding phase space trajectories.

As was shown in Ref. [58], for the case $m=0$, the dynamical system (9]) has the following two fixed points,

$$
\phi_{*}^{1}=\left(x_{1}, x_{2}, x_{3}\right)=(-1,0,2), \phi_{*}^{2}=\left(x_{1}, x_{2}, x_{3}\right)=(0,-1,2),
$$

and as it was shown numerically, for various initial conditions, the fixed point $\phi_{*}^{1}=\left(x_{1}, x_{2}, x_{3}\right)=(-1,0,2)$ is stable and the fixed point $\phi_{*}^{2}\left(x_{1}, x_{2}, x_{3}\right)=(0,-1,2)$ is unstable. Also, by substituting the value of $x_{3}$ corresponding to both fixed points (14), namely $x_{3}=2$, we obtain $w_{\text {eff }}=-1$, so both the fixed points $\phi_{*}^{1}$ and $\phi_{*}^{2}$ are de Sitter fixed points, or equivalently, de Sitter attractors. Moreover, as was shown in Ref. [58], the vacuum $f(R)$ gravity which leads to the fixed point $\phi_{*}^{1}$ has the following functional form,

$$
f(R) \simeq \Lambda_{1}-24 \Lambda_{2} e^{-\frac{R}{24 H_{i}}}
$$

while the functional form of the $f(R)$ gravity which leads to the fixed point $\phi_{*}^{2}=(0,-1,2)$ at leading order, is the following,

$$
f(R) \simeq \alpha R^{2}
$$

Finally, the variables $x_{1}, x_{2}$ and $x_{3}$ (8), satisfy the Friedmann constraint,

$$
x_{1}+x_{2}+x_{3}=1
$$

which is also satisfied by the fixed points. Having discussed the flat vacuum $f(R)$ gravity phase space structure, in the next section we present in detail the effects of spatial curvature on the phase space structure of vacuum $f(R)$ gravity.

\section{AUTONOMOUS VACUUM $f(R)$ GRAVITY DYNAMICAL SYSTEM IN SPATIALLY CURVED SPACETIME}

In this section we shall investigate how it is possible to construct an autonomous dynamical system for a vacuum $f(R)$ gravity theory, for a spatially non-flat FRW metric. The action of the theory and the equations of motion for general metric remain the same as in Eqs. (1) and (3) respectively. So let us assume that the metric has the general non-spatially flat FRW form,

$$
d s^{2}=-d t^{2}+a(t)^{2}\left(\frac{d r^{2}}{1-K r^{2}}+r^{2} d \theta^{2}+r^{2} \sin ^{2} \theta d \varphi^{2}\right)
$$

with $a(t)$ being the scale factor and $K$ is the curvature of the three dimensional spacelike hypersurface $t=$ const, which can be $K=0, \pm 1$. In this case, the Ricci scalar has the following form,

$$
R=6 \dot{H}+12 H^{2}+\frac{6 K}{a^{2}}
$$


where we have used a physical units system in which $\kappa^{2}=8 \pi G=1$, and we adopt this notation for simplicity in the following. For the metric (18), the equations of motion (3) become,

$$
\begin{aligned}
& 3 F H^{2}+\frac{3 K F}{a^{2}}=-\frac{f-F R}{2}-3 H \dot{F}, \\
& \ddot{F}=H \dot{F}-2 F \dot{H}+\frac{2 K F}{a^{2}},
\end{aligned}
$$

where again $F(R)=\frac{\partial f}{\partial R}, F^{\prime}(R)=\frac{\partial F}{\partial R}$, and $F^{\prime \prime}(R)=\frac{\partial^{2} F}{\partial R^{2}}$. Two useful relations for the extraction of the autonomous dynamical system corresponding to the cosmological equations (20) are the following,

$$
\begin{aligned}
& \dot{H}=\frac{R}{6}-2 H^{2}-\frac{K}{a^{2}}, \\
& \dot{R}=24 H \dot{H}+6 \ddot{H}-\frac{12 K H}{a^{2}},
\end{aligned}
$$

which we shall extensively make use of in the following. In order to extract an autonomous dynamical system from Eqs. (20), it is compelling to introduce the following variables (recall we have set $\kappa^{2}=1$ ),

$$
x_{1}=-\frac{\dot{F}(R)}{F(R) H}, \quad x_{2}=-\frac{f(R)}{6 F(R) H^{2}}, \quad x_{3}=\frac{R}{6 H^{2}}, \quad x_{4}=-\frac{K}{a^{2} H^{2}},
$$

so by comparing Eqs. (8) and (22) it can be seen that due to the non-zero curvature effects, it is required to use an additional variable in order to extract an autonomous dynamical system from the $f(R)$ cosmological equations. Again, by using the $e$-foldings number as a dynamical variable, by using the new variables (22) and the cosmological equations (20), we obtain the following autonomous dynamical system for the vacuum $f(R)$ gravity in a spatially non-flat FRW background,

$$
\begin{aligned}
& \frac{\mathrm{d} x_{1}}{\mathrm{~d} N}=-4+3 x_{1}+2 x_{3}-x_{1} x_{3}+x_{1}^{2}+4 x_{4}-x_{1} x_{4} \\
& \frac{\mathrm{d} x_{2}}{\mathrm{~d} N}=8+m-4 x_{3}+x_{2} x_{1}-2 x_{2} x_{3}+4 x_{2}-6 x_{4}-2 x_{2} x_{4}, \\
& \frac{\mathrm{d} x_{3}}{\mathrm{~d} N}=-8-m+8 x_{3}-2 x_{3}^{2}+6 x_{4}-2 x_{3} x_{4} \\
& \frac{\mathrm{d} x_{4}}{\mathrm{~d} N}=-2 x_{3} x_{4}-2 x_{4}^{2}+4 x_{4}-2 x_{4}
\end{aligned}
$$

where we used Eqs. (20), (21), (22) and (10) for the extraction of the dynamical system, and in this case too, the parameter $m$ is given in Eq. (11). As in the flat FRW metric case, the only $N$-dependence of the dynamical system (23) is contained in the parameter $m$, so if we assume that this is constant, then the dynamical system is rendered autonomous. The nature of the possible fixed points of the dynamical system (23) will be determined by the total EoS parameter $w_{e f f}$, which in the non-flat FRW dynamical system case has the following form,

$$
w_{e f f}=-1-\frac{2}{3}\left(x_{3}-2+x_{4}\right)
$$

a relation which easily follows if Eqs. (12), (21) and (22) are combined. Finally, the Friedmann constraint in this case reads,

$$
x_{1}+x_{2}+x_{3}+x_{4}=1
$$

In the following sections we shall investigate the structure of the phase space for the non-flat FRW vacuum $f(R)$ gravity, for various constant values of the parameter $m$, emphasizing on the cases $m=0, m=-\frac{9}{2}$ and $m=-8$, which describe de Sitter, matter and radiation domination fixed points.

\section{A. Study de Sitter Attractors of Spatially Curved $f(R)$ Gravity Phase Space}

Let us focus our study in the case $m=0$, which as we saw in the flat vacuum $f(R)$ gravity corresponds to de Sitter fixed points. So in this section we will address the following questions, are the fixed points of the non-flat vacuum 
$f(R)$ gravity dynamical system (23) de Sitter vacua, and if yes, are these stable, or the presence of the curvature terms destabilizes the phase space? For $m=0$, the dynamical system (23) is rendered autonomous, so the study of the behavior of the trajectories in the phase space can be studied in a easy and formal way, by examining the linearized system. Let us recall the formalism of the Hartman-Grobman theorem, which will be useful in the following sections. Consider a dynamical system of the form,

$$
\frac{\mathrm{d} \Phi}{\mathrm{d} t}=g(\Phi(t))
$$

with $g(\Phi(t))$ being a locally Lipschitz continuous map of the following form $g: R^{n} \rightarrow R^{n}$. Let the fixed points of the autonomous dynamical system (26) be $\phi_{*}$. The Jacobian matrix of the linearized dynamical system near a fixed point, denoted as $\mathcal{J}(g)$, is equal to,

$$
\mathcal{J}=\sum_{i} \sum_{j}\left[\frac{\partial \mathrm{g}_{\mathrm{i}}}{\partial x_{j}}\right]
$$

By calculating the Jacobian at the fixed points, this will eventually reveal whether a fixed point is stable or not, only in the case that the fixed point is hyperbolic. A hyperbolic fixed point corresponds to the case that the eigenvalues of the Jacobian matrix at the fixed point, namely $e_{i}$, have non-zero real parts, that is, $\operatorname{Re}\left(e_{i}\right) \neq 0$. Then the Hartman-Grobman ensures that the linearized dynamical system,

$$
\frac{\mathrm{d} \Phi}{\mathrm{d} t}=\left.\mathcal{J}(g)(\Phi)\right|_{\Phi=\phi_{*}}\left(\Phi-\phi_{*}\right)
$$

is locally homeomorphic to the initial dynamical system (26), at the vicinity of the fixed points $\phi_{*}$, hence by studying the linearized dynamical system is enough in order to understand the behavior of the trajectories near the fixed points, and therefore decide about their stability. If the eigenvalues of the Jacobian satisfy $\operatorname{Re}(\sigma(\mathcal{J}(g)))<0$, then the fixed point is stable, and in all other cases, the fixed point is unstable.

Let us now find the fixed points of the dynamical system (23) in the case $m=0$, and let the functions $f_{i}$ for general $m$, be defined as follows,

$$
\begin{aligned}
& f_{1}\left(x_{1}, x_{2}, x_{3}, x_{4}\right)=-4+3 x_{1}+2 x_{3}-x_{1} x_{3}+x_{1}^{2}+4 x_{4}-x_{1} x_{4}, \\
& f_{2}\left(x_{1}, x_{2}, x_{3}, x_{4}\right)=8+m-4 x_{3}+x_{2} x_{1}-2 x_{2} x_{3}+4 x_{2}-6 x_{4}-2 x_{2} x_{4}, \\
& f_{3}\left(x_{1}, x_{2}, x_{3}, x_{4}\right)=-8-m+8 x_{3}-2 x_{3}^{2}+6 x_{4}-2 x_{3} x_{4}, \\
& f_{4}\left(x_{1}, x_{2}, x_{3}, x_{4}\right)=-2 x_{3} x_{4}-2 x_{4}^{2}+4 x_{4}-2 x_{4},
\end{aligned}
$$

and the corresponding Jacobian matrix,

$$
\mathcal{J}=\sum_{i=1}^{4} \sum_{j=1}^{4}\left[\frac{\partial \mathrm{f}_{\mathrm{i}}}{\partial x_{j}}\right]
$$

is equal to,

$$
\mathcal{J}=\left(\begin{array}{cccc}
2 x_{1}-x_{3}-x_{4}+3 & 0 & 2-x_{1} & 4-x_{1} \\
x_{2} & x_{1}-2 x_{3}-2 x_{4}+4 & -2 x_{2}-4 & -2 x_{2}-6 \\
0 & 0 & -4 x_{3}-2 x_{4}+8 & 6-2 x_{3} \\
0 & 0 & -2 x_{4} & -2 x_{3}-4 x_{4}+2
\end{array}\right)
$$

Focusing now in the case $m=0$, by solving the system of equations $f_{i}=0, i=1, . ., 4$, we obtain the following fixed points with physical significance,

$$
\phi_{*}^{1}=\left(x_{1}, x_{2}, x_{3}, x_{4}\right)=(-1,0,2,0), \phi_{*}^{2}=\left(x_{1}, x_{2}, x_{3}, x_{4}\right)=\left(0, x_{2}, 2,0\right) .
$$

By looking at Eqs. (32) and (14), the similarity is obvious, since the only difference is the existence of the condition $x_{4}=0$ in the new fixed points of the phase space. Also for the fixed point $\phi_{*}^{2}$, the values of $x_{2}$ are free to be chosen, but in order to have consistency with the Friedmann constraint (25), we must require $x_{2}=-1$ for the fixed point $\phi_{*}^{2}$, therefore the resulting fixed points for the case $m=0$ are,

$$
\phi_{*}^{1}=\left(x_{1}, x_{2}, x_{3}, x_{4}\right)=(-1,0,2,0), \phi_{*}^{2}=\left(x_{1}, x_{2}, x_{3}, x_{4}\right)=(0,-1,2,0) .
$$


Clearly, for both the fixed points, the EoS parameter (24) is equal to $w_{\text {eff }}=-1$ when evaluated at the fixed points (33), so both the fixed points (33) are de Sitter fixed points. As we saw in the previous section, for the flat FRW vacuum $f(R)$ gravity case, the fixed point $\phi_{*}^{1}$ was found to be stable, while the fixed point $\phi_{*}^{2}$ was unstable. Let us now see what new features does the variable $x_{4}$ introduces in the phase space. In order to see this, let us calculate the eigenvalues of the Jacobian at the fixed points, so we start off with $\phi_{*}^{1}$, so the Jacobian in this case is,

$$
\mathcal{J}=\left(\begin{array}{cccc}
-1 & 0 & 3 & 5 \\
0 & -1 & -4 & -6 \\
0 & 0 & 0 & 2 \\
0 & 0 & 0 & -2
\end{array}\right)
$$

and the corresponding eigenvalues are $(-2,-1,-1,0)$, while for the fixed point $\phi_{*}^{2}$, the Jacobian is,

$$
\mathcal{J}=\left(\begin{array}{cccc}
1 & 0 & 2 & 4 \\
-1 & 0 & -2 & -4 \\
0 & 0 & 0 & 2 \\
0 & 0 & 0 & -2
\end{array}\right)
$$

with eigenvalues $(-2,1,0,0)$. Therefore, by looking the eigenvalues it is obvious that both fixed point are not hyperbolic, and in effect, numerical analysis is needed to reveal the stability of the fixed points. After a thorough investigation of the trajectories in the phase space spanned by the variables $x_{1}, x_{2}, x_{3}$ and $x_{4}$, for various initial conditions, the resulting picture is that the trajectories of all the variables blow-up after a few $e$-foldings, so in effect, the two fixed points (33) are strongly unstable. This can also be seen in Fig. 1, where we plotted the behavior of $x_{1}(N), x_{2}(N), x_{3}(N)$ and $x_{4}(N)$ as functions of the $e$-foldings. As it can be seen in Fig. 1 the trajectories blow-up
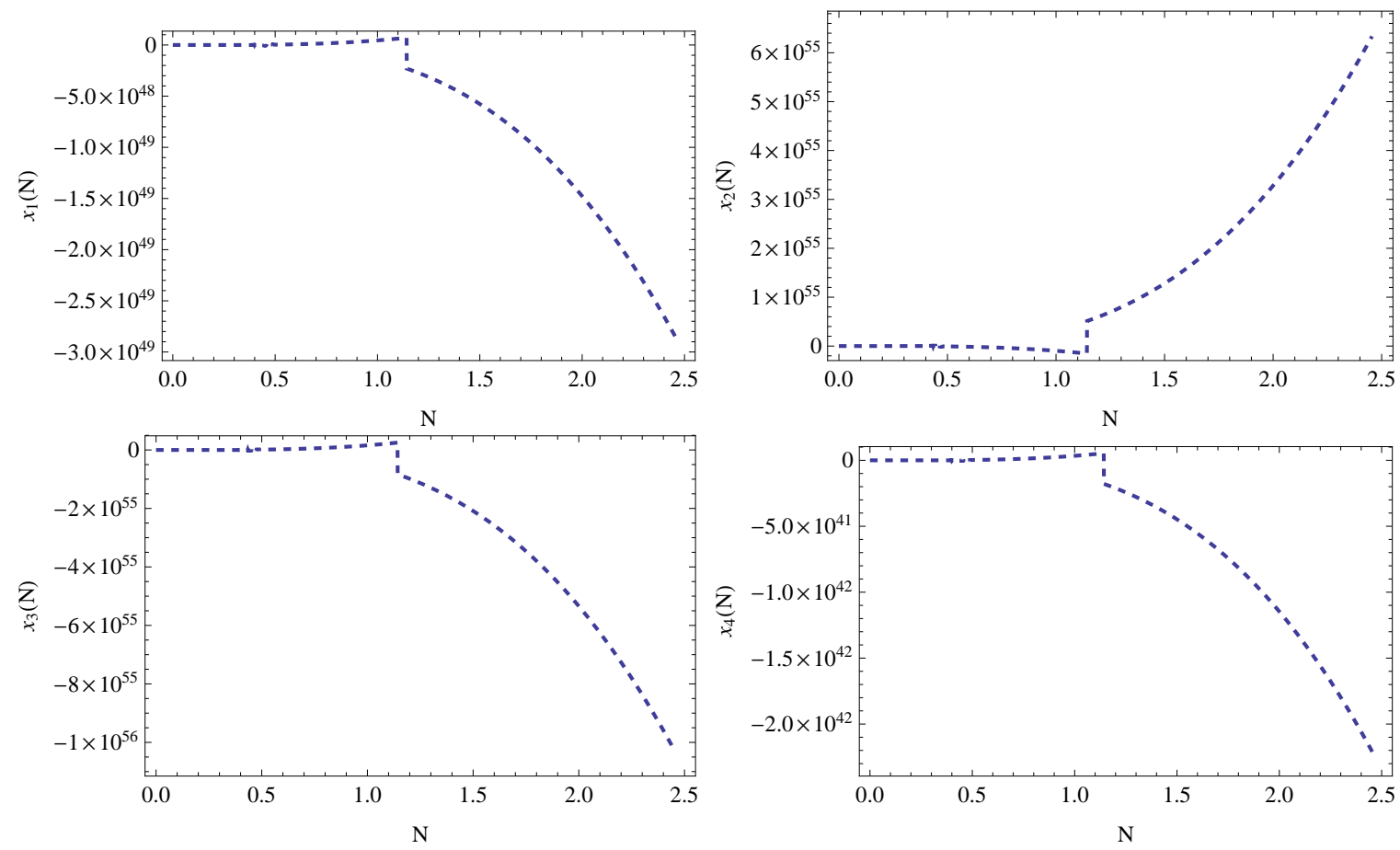

FIG. 1: The behavior of $x_{1}(N), x_{2}(N), x_{3}(N)$ and $x_{4}(N)$ as functions of the e-foldings, for the non-flat FRW autonomous vacuum $f(R)$ gravity dynamical system in the case $m=0$.

even after a few $e$-foldings, and this occurs for various initial conditions, for both positive and negative curvatures, even for small initial values. Hence, we can conclude that the effect of spatial curvature in the vacuum $f(R)$ gravity phase space, is that it completely destabilizes the stable de Sitter fixed points of the flat case. The result is model independent and covers all possible cases of $f(R)$ gravity that may lead to de Sitter vacua. This can also be seen for various initial conditions in Fig. 2, where we plotted the trajectories in the $x_{3}-x_{4}$ plane. As it can be seen, there exist multiple infinite trajectories, a fact that depicts the strong instabilities that the curvature introduces in the phase space. Before closing it is worth noting that in this case too, the functional form of the $f(R)$ gravity leading to 


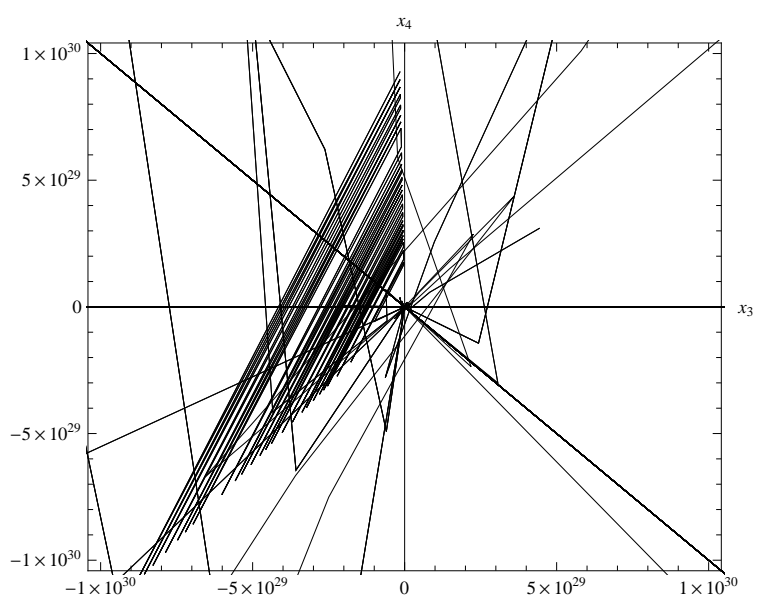

FIG. 2: The trajectories in the $x_{3}-x_{4}$ plane for various initial conditions, for the non-flat FRW autonomous vacuum $f(R)$ gravity dynamical system in the case $m=0$.

the fixed points $\phi_{*}^{1}$ and $\phi_{*}^{2}$ (33), are given in Eqs. (15) and (16) respectively. More importantly, both the fixed points (33) have $x_{4}=0$. Thus the dynamical system has essentially the same fixed points with the flat case $f(R)$ gravity, however the effect of the curvature is non-trivial in the dynamical system, since it destabilizes the fixed points. The dynamical system of the non-flat case obviously has a rich underlying structure, possibly in the form of some unstable manifolds, the study of which is beyond the scopes of this article.

Before closing we need to discuss an important issue having to do with the dimensions of the dynamical system (23) and the Friedman constraint (25). Basically one could reduce the dimensions of the dynamical system to three instead of four by taking into account the Friedmann constraint, however we kept all the variables in order to have a concrete idea on how these behave as functions of the $e$-foldings number, and see how the instability occurs in the system. In the case at hand, the presence of the $x_{4}$ terms make the system strongly unstable, so there surely is some unstable submanifold related to $x_{4}$. We hope to address these mathematical issues in a focused future work.

\section{B. Study of Matter and Radiation Domination Era Attractors of Spatially Curved $f(R)$ Gravity Phase Space}

Now let us turn our focus on other cosmological solutions, so let us discuss the case $m=-\frac{9}{2}$, which as was shown in Ref. [58], corresponds to matter domination fixed points of the autonomous dynamical system, with $w_{\text {eff }}=0$. The fixed points of the corresponding flat autonomous dynamical system (9) with $m=-\frac{9}{2}$, are the following [58],

$$
\phi_{*}^{1}=\left(\frac{1}{4}(-5-\sqrt{73}), \frac{1}{4}(7+\sqrt{73}), \frac{1}{2}\right), \quad \phi_{*}^{2}=\left(\frac{1}{4}(\sqrt{73}-5), \frac{1}{4}(7-\sqrt{73}), \frac{1}{2}\right) .
$$

The eigenvalues of the Jacobian matrix $\mathcal{J}$ for the first fixed point $\phi_{*}^{1}$ are $(6,-4.272,-0.386001)$, while for the fixed point $\phi_{*}^{2}$ these are $(6,4.272,3.886)$. In effect, both equilibria are hyperbolic and unstable. Also, since $x_{3}=\frac{1}{2}$, for both the fixed points (36), in both cases the EoS parameter is $w_{\text {eff }}=0$, and this justifies why these fixed points are called matter domination fixed points.

Having discussed the flat autonomous $f(R)$ gravity dynamical system for the case $m=-\frac{9}{2}$, let us now see the effects of the spatial curvature on the corresponding autonomous dynamical system (23). For $m=-\frac{9}{2}$ the physically acceptable fixed points of the dynamical system are the following,

$$
\begin{aligned}
& \phi_{*}^{1}=\left(x_{1}, x_{2}, x_{3}, x_{4}\right)=\left(\frac{1}{4}(-5-\sqrt{73}), \frac{1}{2}\left(\frac{1}{2}(5+\sqrt{73})+1\right), \frac{1}{2}, 0\right), \\
& \phi_{*}^{2}=\left(x_{1}, x_{2}, x_{3}, x_{4}\right)=\left(\frac{1}{4}(\sqrt{73}-5), \frac{1}{2}\left(\frac{1}{2}(5-\sqrt{73})+1\right), \frac{1}{2}, 0\right) .
\end{aligned}
$$

As it can be easily checked, for both the fixed points $\phi_{*}^{1}$ and $\phi_{*}^{2}$, the EoS parameter (24) becomes $w_{\text {eff }}=0$, so both the fixed points (37) are matter domination fixed points. Also the Friedmann constraint (25) is also satisfied for both 
the fixed points. For the fixed point $\phi_{*}^{1}$ the Jacobian matrix (31) becomes,

$$
\mathcal{J}=\left(\begin{array}{cccc}
-\frac{\sqrt{73}}{2} & 0 & \frac{1}{4}(13+\sqrt{73}) & \frac{1}{4}(21+\sqrt{73}) \\
\frac{1}{4}(7+\sqrt{73}) & \frac{1}{4}(7-\sqrt{73}) & \frac{1}{2}(-15-\sqrt{73}) & \frac{1}{2}(-19-\sqrt{73}) \\
0 & 0 & 6 & 5 \\
0 & 0 & 0 & 1
\end{array}\right)
$$

and it's eigenvalues are $(6,-4.272,1,-0.386001)$. In effect, the fixed point $\phi_{*}^{1}$ is a hyperbolic and unstable fixed point. Accordingly, for the fixed point $\phi_{*}^{2}$, the Jacobian matrix is,

$$
\mathcal{J}=\left(\begin{array}{cccc}
\frac{\sqrt{73}}{2} & 0 & \frac{1}{4}(13-\sqrt{73}) & \frac{1}{4}(21-\sqrt{73}) \\
\frac{1}{4}(7-\sqrt{73}) & \frac{1}{4}(7+\sqrt{73}) & \frac{1}{2}(-15+\sqrt{73}) & \frac{1}{2}(-19+\sqrt{73}) \\
0 & 0 & 6 & 5 \\
0 & 0 & 0 & 1
\end{array}\right)
$$

and the corresponding eigenvalues are $(6,4.272,3.886,1)$, so the fixed point $\phi_{*}^{2}$ is also hyperbolic and unstable. The instability of the phase space can also be seen in Fig. 3. where we plot the functional dependence of the variables $x_{i}(N), i=1, . ., 4$, by solving numerically the dynamical system equations.
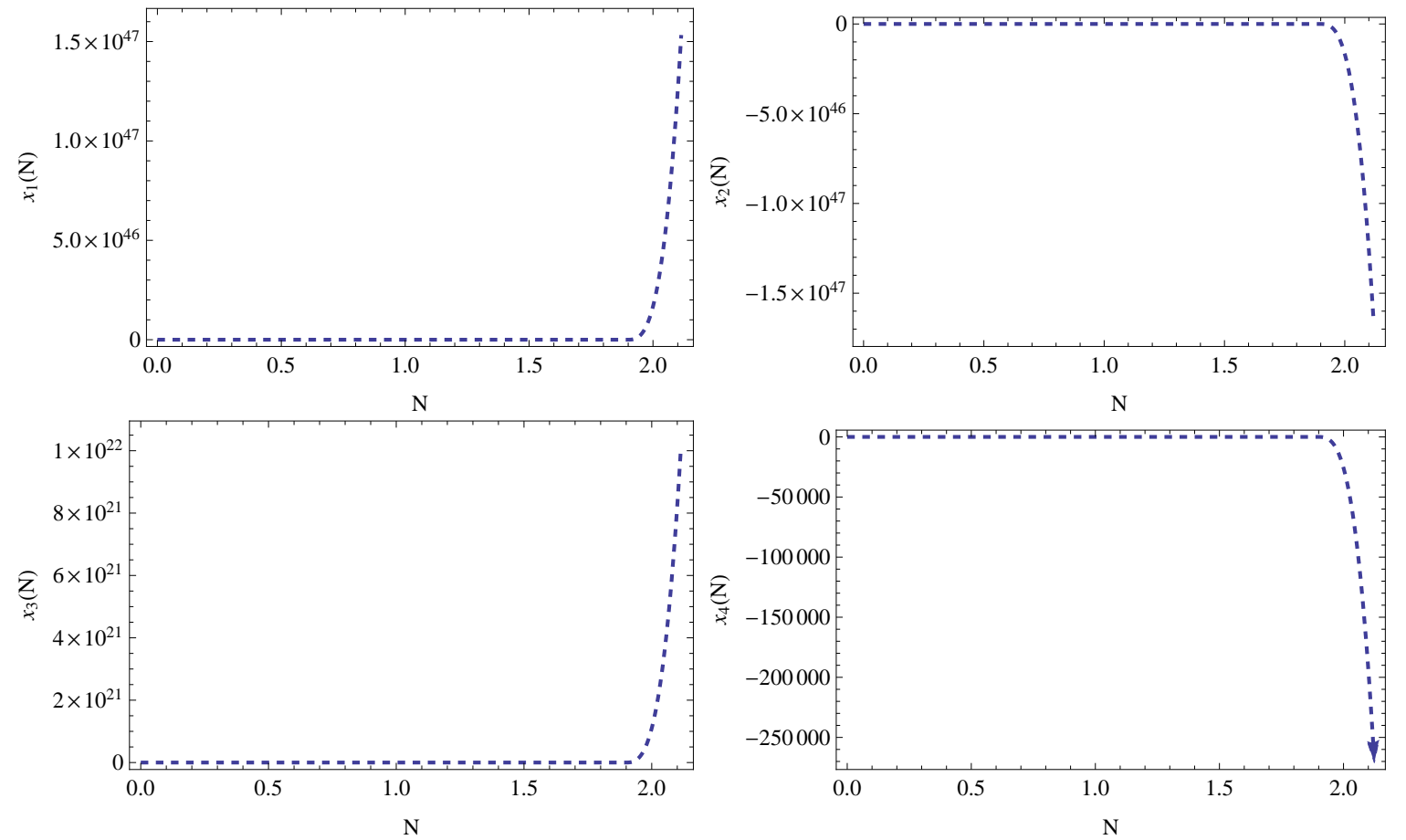

FIG. 3: The behavior of $x_{1}(N), x_{2}(N), x_{3}(N)$ and $x_{4}(N)$ as functions of the e-foldings, for the non-flat FRW autonomous vacuum $f(R)$ gravity dynamical system in the case $m=-\frac{9}{2}$.

Similar considerations can be made for radiation domination fixed points. By choosing $m=-8$, the fixed points of the dynamical system (23) are the following,

$$
\begin{aligned}
& \phi_{*}^{1}=\left(x_{1}, x_{2}, x_{3}, x_{4}\right)=(-4,5,0,0), \\
& \phi_{*}^{2}=\left(x_{1}, x_{2}, x_{3}, x_{4}\right)=(1,0,0,0) .
\end{aligned}
$$

For both the fixed points $\phi_{*}^{1}$ and $\phi_{*}^{2}$, the EoS parameter (24) becomes $w_{\text {eff }}=\frac{1}{3}$, so both the fixed points (40) are radiation domination fixed points. For the fixed point $\phi_{*}^{1}$ the Jacobian matrix (31) is equal to,

$$
\mathcal{J}=\left(\begin{array}{cccc}
-5 & 0 & 6 & 8 \\
5 & 0 & -14 & -16 \\
0 & 0 & 8 & 6 \\
0 & 0 & 0 & 2
\end{array}\right)
$$


the eigenvalues of which are $(8,-5,2,0)$. Also for the fixed point $\phi_{*}^{2}$, the Jacobian matrix is,

$$
\mathcal{J}=\left(\begin{array}{cccc}
5 & 0 & 1 & 3 \\
0 & 5 & -4 & -6 \\
0 & 0 & 8 & 6 \\
0 & 0 & 0 & 2
\end{array}\right)
$$

and the eigenvalues are $(8,5,5,2)$, so only the fixed point $\phi_{*}^{2}$ is hyperbolic and it is clearly unstable. Finally, a numerical analysis of the phase space indicates that strong instabilities occur in the phase space.

In conclusion, apart from the de Sitter fixed points studied in the previous section, also the matter and radiation domination fixed points are unstable equilibria of the autonomous curved FRW vacuum $f(R)$ gravity dynamical system. Hence, this result supports our claim that the effect of curvature is to destabilize the phase space structure of vacuum $f(R)$ gravity.

Qualitatively, our results clearly indicate that the stable de Sitter attractors of the flat spacetime vacuum $f(R)$ gravity are destabilized strongly even in the presence of a small non-zero curvature in the metric. This result holds true even for positive (elliptic spatial sections) and negative curvatures (hyperbolic spatial curvatures). Hence due to the unstable phase space structure, we have strong hints to claim that inflation in the non-flat vacuum $f(R)$ gravity theories is problematic or at least is not so universal, compared to the flat case one, since there is no stable de Sitter attractor in the theory. The existence of stable inflationary attractors in a gravitational theory is of fundamental importance, since even the slow-roll expansion assumes a stable attractor that eventually will attract all the phase space trajectories on it. An insightful work analyzing the slow-roll expansion in view of the existence of inflationary attractors is [70].

\section{CONCLUSIONS}

In this paper we studied the effects of spatial curvature in the autonomous dynamical system of vacuum $f(R)$ gravity. After appropriately choosing the variables, we constructed a dynamical system which can be rendered autonomous in the case that the parameter $m=-\frac{\ddot{H}}{H^{3}}$ takes constant values. We focused on three cases of interest, namely for $m=0$,

$m=-\frac{9}{2}$ and $m=-8$. The case $m=0$ corresponds to de Sitter vacua, since the fixed points have a EoS parameter equal to $w_{\text {eff }}=-1$, which is characteristic of the de Sitter evolution. As we demonstrated, the presence of spatial curvature destabilizes the flat space phase space, which was shown in a previous work to be stable. The result is robust towards the choice of different initial conditions. The absence of stable inflationary attractors indicates that inflation in non-flat vacuum $f(R)$ gravity is problematic and not so universal compared with the flat vacuum $f(R)$ gravity case. The same instability behavior occurs for the case $m=-\frac{9}{2}$, which corresponds to matter domination related fixed points, and also the same behavior applies in the case $m=-8$, which describes radiation domination equilibria. As we demonstrated, all the matter and radiation domination fixed points are unstable hyperbolic equilibria. However, the flat case autonomous dynamical system for $f(R)$ gravity, in the cases of matter and radiation domination equilibria, was also unstable, so the most interesting case of this study was the de Sitter case, and particularly one needs to understand why the non-flat autonomous dynamical system becomes destabilized. It is clear that the new variable $x_{4}$, which was absent in the flat case, brings some instability in the dynamical system. So it is certain that the phase space of the non-flat de Sitter case has a rich underlying mathematical structure. It is highly possible that non-stable manifolds are formed, strongly related with the $x_{4}$ subspace. The study of these unstable manifolds is mathematically non-trivial because the dynamical system is highly non-linear, but the complete investigation of the above issues will be given elsewhere. Finally, it would be interesting to extend the current study by taking into account dark matter and baryonic matter. Eventually, in this case the dynamical system would become much more complicated and only numerical investigation would be the only approach for studying the dynamical evolution of the trajectories in the phase space. 


\section{Acknowledgments}

This work is supported by MINECO (Spain), FIS2016-76363-P (S.D.O), by project 2017 SGR247 (AGAUR, Catalonia) (S.D. Odintsov).

[1] S. Capozziello, M. De Laurentis, Phys. Rept. 509, 167 (2011);

V. Faraoni and S. Capozziello, Fundam. Theor. Phys. 170 (2010). doi:10.1007/978-94-007-0165-6

[2] G. J. Olmo, Int. J. Mod. Phys. D 20 (2011) 413 doi:10.1142/S0218271811018925 arXiv:1101.3864 [gr-qc]].

[3] S. Nojiri, S. D. Odintsov and V. K. Oikonomou, Phys. Rept. 692 (2017) 1 doi:10.1016/j.physrep.2017.06.001 arXiv:1705.11098 [gr-qc]].

[4] S. Nojiri, S.D. Odintsov, Phys. Rept. 505, 59 (2011);

[5] S. Nojiri, S.D. Odintsov, eConf C0602061, 06 (2006) [Int. J. Geom. Meth. Mod. Phys. 4, 115 (2007)].

[6] A. de la Cruz-Dombriz and D. Saez-Gomez, Entropy 14 (2012) 1717 doi:10.3390/e14091717 arXiv:1207.2663 [gr-qc]].

[7] S. Nojiri and S. D. Odintsov, Phys. Rev. D 68 (2003) 123512 doi:10.1103/PhysRevD.68.123512 hep-th/0307288.

[8] S. Nojiri and S. D. Odintsov, Phys. Rev. D 74 (2006) 086005 doi:10.1103/PhysRevD.74.086005 hep-th/0608008.

[9] S. Capozziello, S. Nojiri, S.D. Odintsov, A. Troisi, Phys.Lett. B639 (2006) 135 astro-ph/0604431; S. Nojiri, S. D. Odintsov, Phys.Rev. D77 (2008) 026007 arXiv:0710.1738

[10] S. Capozziello, V.F. Cardone, S. Carloni, A. Troisi, Int.J.Mod.Phys. D12 (2003) 1969 astro-ph/0307018

[11] W. Hu, I. Sawicki, Phys.Rev.D76 (2007) 064004 arXiv:0705.1158

[12] S. M. Carroll, V. Duvvuri, M. Trodden, M. S. Turner, Phys.Rev. D70 (2004) 043528 astro-ph/0306438; S. Capozziello, Int.J.Mod.Phys.D11, 483 (2002) gr-qc/0201033

[13] O. Bertolami, R. Rosenfeld, Int.J.Mod.Phys. A23 (2008) 4817 arXiv:0708.1784.

[14] A. Capolupo, S. Capozziello, G. Vitiello, Int.J.Mod.Phys. A23 (2008) 4979 arXiv:0705.0319.

[15] P. K.S. Dunsby, E. Elizalde, R. Goswami, S. Odintsov, D. S. Gomez, Phys.Rev. D82 (2010) 023519 arXiv:1005.2205

[16] G. Cognola, E. Elizalde, S. Nojiri, S.D. Odintsov, L. Sebastiani, S. Zerbini, Phys.Rev. D77 (2008) 046009 arXiv:0712.4017 ]

[17] K. Bamba, Chao-Qiang Geng, Chung-Chi Lee, JCAP 1008 (2010) 021 arXiv:1005.4574.

[18] S. Nojiri, S. D. Odintsov, D. Saez-Gomez, Phys.Lett. B681 (2009) 74 arXiv:0908.1269.

[19] S. Capozziello, V. F. Cardone, A. Troisi, Phys.Rev. D71 (2005) 043503 astro-ph/0501426.

[20] J. C.C. de Souza, Valerio Faraoni, Class.Quant.Grav. 24 (2007) 3637 arXiv:0706.1223; V. Faraoni, Phys.Rev. D74 (2006) 104017 astro-ph/0610734;G. J. Olmo, Phys.Rev.Lett. 95 (2005) 261102 gr-qc/0505101; G. J. Olmo, Phys.Rev. D75 (2007) 023511 gr-qc/0612047

[21] K. Bamba, S. Nojiri and S. D. Odintsov, JCAP 0810 (2008) 045 doi:10.1088/1475-7516/2008/10/045 arXiv:0807.2575 [hep-th]].

[22] S. A. Appleby, R. A. Battye, A. A. Starobinsky, JCAP 1006 (2010) 005 arXiv:0909.1737.

[23] S. A. Appleby, R. A. Battye, Phys.Lett.B654 (2007) 7 arXiv:0705.3199; S. A. Appleby, R. A. Battye, JCAP 0805 (2008) 019 arXiv:0803.1081]

[24] V. Faraoni, Phys.Rev. D75 (2007) 067302 gr-qc/0703044

[25] Dynamical Systems in Cosmology, J. Wainwright, G. F. R. Ellis, Cambridge University Press, 2009

[26] S. Bahamonde, C. G. Boehmer, S. Carloni, E. J. Copeland, W. Fang and N. Tamanini, arXiv:1712.03107 [gr-qc].

[27] C. G. Boehmer and N. Chan, doi:10.1142/9781786341044.0004 arXiv:1409.5585 [gr-qc].

[28] S. Carloni, J. L. Rosa and J. P. S. Lemos, arXiv:1808.07316] [gr-qc].

[29] C. G. Boehmer, T. Harko and S. V. Sabau, Adv. Theor. Math. Phys. 16 (2012) no.4, 1145 doi:10.4310/ATMP.2012.v16.n4.a2 [arXiv:1010.5464 [math-ph]].

[30] N. Goheer, J. A. Leach and P. K. S. Dunsby, Class. Quant. Grav. 24 (2007) 5689 doi:10.1088/0264-9381/24/22/026 arXiv:0710.0814 [gr-qc]].

[31] G. Leon and E. N. Saridakis, JCAP 1504 (2015) no.04, 031 doi:10.1088/1475-7516/2015/04/031 arXiv:1501.00488 [gr-qc]].

[32] G. Leon and E. N. Saridakis, Class. Quant. Grav. 28 (2011) 065008 doi:10.1088/0264-9381/28/6/065008 arXiv:1007.3956 $[\mathrm{gr}-\mathrm{qc}]]$.

[33] J. C. C. de Souza and V. Faraoni, Class. Quant. Grav. 24 (2007) 3637 doi:10.1088/0264-9381/24/14/006 arXiv:0706.1223 [gr-qc]].

[34] A. Giacomini, S. Jamal, G. Leon, A. Paliathanasis and J. Saavedra, Phys. Rev. D 95 (2017) no.12, 124060 doi:10.1103/PhysRevD.95.124060 arXiv:1703.05860 [gr-qc]].

[35] G. Kofinas, G. Leon and E. N. Saridakis, Class. Quant. Grav. 31 (2014) 175011 doi:10.1088/0264-9381/31/17/175011 arXiv:1404.7100 [gr-qc]].

[36] G. Leon and E. N. Saridakis, JCAP 1303 (2013) 025 doi:10.1088/1475-7516/2013/03/025 arXiv:1211.3088 [astro-ph.CO]].

[37] T. Gonzalez, G. Leon and I. Quiros, Class. Quant. Grav. 23 (2006) 3165 doi:10.1088/0264-9381/23/9/025 astro-ph/0702227.

[38] A. Alho, S. Carloni and C. Uggla, JCAP 1608 (2016) no.08, 064 doi:10.1088/1475-7516/2016/08/064 arXiv:1607.05715 $[\mathrm{gr}-\mathrm{qc}]]$. 
[39] S. K. Biswas and S. Chakraborty, Int. J. Mod. Phys. D 24 (2015) no.07, 1550046 doi:10.1142/S0218271815500467 arXiv:1504.02431 [gr-qc]].

[40] D. Muller, V. C. de Andrade, C. Maia, M. J. Reboucas and A. F. F. Teixeira, Eur. Phys. J. C 75 (2015) no.1, 13 doi:10.1140/epjc/s10052-014-3227-2 arXiv:1405.0768 [astro-ph.CO]].

[41] B. Mirza and F. Oboudiat, Int. J. Geom. Meth. Mod. Phys. 13 (2016) no.09, 1650108 doi:10.1142/S0219887816501085 arXiv:1412.6640 [gr-qc]].

[42] S. Rippl, H. van Elst, R. K. Tavakol and D. Taylor, Gen. Rel. Grav. 28 (1996) 193 doi:10.1007/BF02105423 gr-qc/9511010.

[43] M. M. Ivanov and A. V. Toporensky, Grav. Cosmol. 18 (2012) 43 doi:10.1134/S0202289312010100 [arXiv:1106.5179 [gr-qc]].

[44] M. Khurshudyan, Int. J. Geom. Meth. Mod. Phys. 14 (2016) no.03, 1750041. doi:10.1142/S0219887817500414

[45] R. D. Boko, M. J. S. Houndjo and J. Tossa, Int. J. Mod. Phys. D 25 (2016) no.10, 1650098 doi:10.1142/S021827181650098X arXiv:1605.03404 [gr-qc]].

[46] S. D. Odintsov, V. K. Oikonomou and P. V. Tretyakov, Phys. Rev. D 96 (2017) no.4, 044022 doi:10.1103/PhysRevD.96.044022 arXiv:1707.08661 [gr-qc]].

[47] L. N. Granda and D. F. Jimenez, arXiv:1710.07273 [gr-qc].

[48] F. F. Bernardi and R. G. Landim, Eur. Phys. J. C 77 (2017) no.5, 290 doi:10.1140/epjc/s10052-017-4858-x arXiv:1607.03506 [gr-qc]].

[49] R. C. G. Landim, Eur. Phys. J. C 76 (2016) no.1, 31 doi:10.1140/epjc/s10052-016-3894-2 arXiv:1507.00902 [gr-qc]].

[50] R. C. G. Landim, Eur. Phys. J. C 76 (2016) no.9, 480 doi:10.1140/epjc/s10052-016-4328-x [arXiv:1605.03550] [hep-th]].

[51] P. Bari, K. Bhattacharya and S. Chakraborty, arXiv:1805.06673 [gr-qc].

[52] S. Chakraborty, arXiv:1805.03237 [gr-qc].

[53] M. G. Ganiou, P. H. Logbo, M. J. S. Houndjo and J. Tossa, arXiv:1805.00332 [gr-qc].

[54] P. Shah, G. C. Samanta and S. Capozziello, arXiv:1803.09247 [gr-qc].

[55] S. D. Odintsov and V. K. Oikonomou, Phys. Rev. D 98 (2018) no.2, 024013 doi:10.1103/PhysRevD.98.024013 arXiv:1806.07295 [gr-qc]].

[56] S. D. Odintsov and V. K. Oikonomou, Phys. Rev. D 97 (2018) no.12, 124042 doi:10.1103/PhysRevD.97.124042 arXiv:1806.01588 [gr-qc]].

[57] V. K. Oikonomou, Int. J. Mod. Phys. D 27 (2018) no.05, 1850059 doi:10.1142/S0218271818500591 arXiv:1711.03389 [gr-qc]].

[58] S. D. Odintsov and V. K. Oikonomou, Phys. Rev. D 96 (2017) no.10, 104049 doi:10.1103/PhysRevD.96.104049 arXiv:1711.02230 [gr-qc]].

[59] K. Kleidis and V. K. Oikonomou, arXiv:1808.04674 [gr-qc].

[60] K. Kleidis and V. K. Oikonomou, Int. J. Geom. Meth. Mod. Phys. 15 (2018) no.08, 1850137 doi:10.1142/S0219887818501372 arXiv:1803.10748 [gr-qc]].

[61] S. D. Odintsov and V. K. Oikonomou, Phys. Rev. D 93 (2016) no.2, 023517 doi:10.1103/PhysRevD.93.023517 arXiv:1511.04559 [gr-qc]].

[62] J. Dutta, W. Khyllep, E. N. Saridakis, N. Tamanini and S. Vagnozzi, JCAP 1802 (2018) 041 doi:10.1088/14757516/2018/02/041 arXiv:1711.07290 [gr-qc]].

[63] M. Gosenca and P. Coles, The Open Journal of Astrophysics, 2016 doi:10.21105/astro.1502.04020 arXiv:1502.04020 [gr$\mathrm{qc}]]$.

[64] R. J. van den Hoogen, A. A. Coley and D. Wands, Class. Quant. Grav. 16 (1999) 1843 doi:10.1088/0264-9381/16/6/317 gr-qc/9901014.

[65] J. Perez, A. Fozfa, T. Carletti, L. Milot and L. Guedezounme, Gen. Rel. Grav. 46 (2014) 1753 doi:10.1007/s10714-0141753-8 arXiv:1306.1037 [gr-qc]].

[66] M. Goliath and G. F. R. Ellis, Phys. Rev. D 60 (1999) 023502 doi:10.1103/PhysRevD.60.023502 gr-qc/9811068.

[67] Stephen Wiggins, Introduction to Applied Nonlinear Dynamical Systems and Chaos, Springer, New York, 2003

[68] J. Ooba, B. Ratra and N. Sugiyama, arXiv:1802.05571 [astro-ph.CO].

[69] J. Ooba, B. Ratra and N. Sugiyama, arXiv:1712.08617 [astro-ph.CO].

[70] A. R. Liddle, P. Parsons and J. D. Barrow, Phys. Rev. D 50 (1994) 7222 doi:10.1103/PhysRevD.50.7222 astro-ph/9408015. 